\title{
Tactile perception of thermal diffusivity
}

\author{
Wouter M. Bergmann Tiest And Astrid M. L. Kappers \\ Utrecht University, Utrecht, The Netherlands
}

\begin{abstract}
The thermal diffusivity of an object is a parameter that controls the rate at which heat is extracted from the hand when it touches that object. It is an important feature for distinguishing materials by means of touch. In order to quantitatively describe the ability of human observers to discriminate between materials on the basis of heat extraction rate, we conducted an experiment in which this heat extraction was performed in a controlled way. In different conditions, subjects were repeatedly asked to select from two stimuli the one that cooled faster. The discrimination threshold was around $43 \%$ of the extraction rate. A rate that was twice as slow also yielded twice the absolute threshold. When we halved the temperature difference between the beginning and end of the stimulus, the threshold did not change as much. In separate experiments, we investigated the different cues that were available in the stimulus: initial cooling rate and end temperature. Both cues were used for discrimination, but cooling rate seemed to be the most important.
\end{abstract}

The thermal diffusivity of a material describes the way heat spreads through it. It is an aspect of materials that is very important for discriminating between them by touch. A wooden and a metal surface with exactly the same surface texture are easily distinguished by touch just on the basis of their different thermal behavior. A material with a high thermal diffusivity conducts heat away quickly upon touch and therefore feels cold. Conversely, a material with a low thermal diffusivity will feel warm to the touch. What is often perceived as "warm" or "cold" has little to do with temperature but is, in fact, caused by different rates of heat extraction. Walking around in air of $10^{\circ} \mathrm{C}$ is a very different thermal experience from swimming in water of $10^{\circ} \mathrm{C}$. This effect is very well known in daily life, and it is therefore surprising that so little is known about the tactile perception of heat extraction rate.

At the basis of heat extraction perception lies temperature sensation. There is evidence that temperature sensation is mediated by separate channels for warm and cold. Both warm and cold temperature receptor populations have been found to include $\mathrm{A} \delta$ and $\mathrm{C}$ type fibers (Spray, 1986). Differences in responses to cold and warm suggest that these stimuli are processed separately (Greenspan, Roy, Caldwell, \& Farooq, 2003). Phenomena like synthetic heat and paradoxical cold suggest that warm, cold, and pain are signaled by both separate fibers and nonspecific multimodal fibers (Green, 2004).

Although equipped with these warm- and coldsensitive systems, humans are not very good at judgments of absolute temperature, as compared with judgments of temperature differences. The reason for this is thought to be that there is no fixed reference point for temperature perception, which is due to adaptation. Adaptation occurs when the hand is immersed in, for example, water or air of a specific temperature. Often, the perceived temperature of an object depends on the temperature difference between adaptation temperature and object temperature, rather than on the absolute temperature (Tritsch, 1988). Indeed, people perform better at the perception of temperature differences; depending on the temperature at which a measurement takes place, the threshold for detecting a difference between the two hands varies from $0.5^{\circ} \mathrm{C}$ to $5.2^{\circ} \mathrm{C}$ (Abbott, 1914). People are even more sensitive to changes in temperature at a single location on the body. With a rate of change of $0.1^{\circ} \mathrm{C} / \mathrm{sec}$ or faster, the cool threshold is between $0.1^{\circ} \mathrm{C}$ and $0.3^{\circ} \mathrm{C}$ (Kenshalo, Holmes, \& Wood, 1968). With slower rates of change, the threshold goes up. Still better is the discrimination between two subsequent temperature changes or cooling pulses: The threshold ranges from $0.03^{\circ} \mathrm{C}$ to $0.06^{\circ} \mathrm{C}$ for different intensities of the cooling pulses on the thenar eminence (Johnson, Darian-Smith, \& LaMotte, 1973). These thresholds were determined using either temperature-controlled water baths or Peltier elements. Incidentally, thresholds differ appreciably over the body surface and go up with age (Stevens \& Choo, 1998). The lips are the most sensitive, but (due to practical or hygienic considerations) most measurements have been taken on the fingers or the thenar eminence. Thresholds at those locations are not much higher, especially in the younger age group (Stevens \& Choo, 1998). Measuring these thresholds can aid in the diagnosis of small fiber neuropathy, although there is no consensus on the exact method to use (Hilz, Glorius, \& Berić, 1995).

Given this high sensitivity to cooling of the skin, the temperature sense can be used to discriminate between objects of the same temperature but with different thermal properties. This is because we are able to accurately 
perceive not only temperature change, as was discussed above, but also heat extraction. Materials with different thermal parameters extract heat at different rates when touched. Katz (1925) reported some experiments with 11 different materials that were ordered from "colder" to "warmer." He found a well-reproduced order among subjects. Discrimination between materials was more difficult if their temperature was close to skin temperature. When their temperature was raised above skin temperature, the perceived order of "warmer" and "colder" reversed. Evidently, the perception of "coldness" is very dependent on the amount of heat flow from the hand to the material or vice versa (Bergmann Tiest \& Kappers, 2008). This human ability to discriminate between different heat extraction rates can be used for diagnostic purposes (Dyck, Curtis, Bushek, \& Offord, 1974). Using a set of four disks of different materials and thicknesses, it was found that the discrimination threshold approximately lies at a difference of an order of magnitude in (initial) heat transfer rate. Further efforts to precisely measure the heat extraction discrimination threshold have thus far been limited. Ino et al. (1993) showed that some materials (aluminum, wood) are more easily identified than others, but they did not relate this to thermal parameters of the materials. In another experiment with 8 different materials, a difference in thermal conductivity of the order of $70 \%$ was needed for discrimination, but other thermal parameters, such as heat capacity, were not taken into account (Jones \& Berris, 2002). Thermal conductivity is the amount of heat per second that flows from one location in a material to another with a given temperature difference between the locations. Later, on the basis of an experiment with 5 different materials, the authors suggested that heat capacity might be the most relevant thermal parameter (Jones \& Berris, 2003). Heat capacity is the amount of heat needed to increase the temperature of a material by a given amount. In this study, only 1 material could be reliably discriminated from the others; a difference of a factor of four in heat capacity was necessary for discrimination. These findings were expanded by Ho and Jones (2004, 2006), using 6 materials that had a better spacing in terms of thermal parameters. In this experiment, materials could be correctly discriminated from each other when their contact coefficients differed by a factor of three or more. The contact coefficient depends on the product of heat capacity and thermal conductivity and determines the intermediate temperature that is initially established when a warmer surface (hand) touches a colder surface (material).

The experiments discussed so far have estimated thresholds of different thermal parameters (initial heat transfer rate, thermal conductivity, heat capacity, contact coefficient). However, due to the spacing of the stimuli, the precision of these estimates has been limited. The difficulty with obtaining precise discrimination thresholds with real materials is that a sufficiently dense and regular spacing of stimuli might not be available. This can be resolved by artificially extracting heat from the fingerfor example, by means of a Peltier heat pump. Ino et al. (1993) have shown, with an experiment involving five ma- terials, that artificially generated temperature sensations can be identified just as well as those provided by real materials. Caldwell and Gosney (1993) also used a Peltier element to simulate thermal sensations in a "tele-taction" context. They showed that subjects could successfully discriminate between four simulated materials. In these two experiments, a prerecorded thermal transient was replayed to the subjects, resulting in a dynamic stimulus. In the experiment by Ho and Jones (2004), a static stimulus was used. Here, the Peltier element was maintained at the initial contact temperature that would be established given the contact coefficient of the simulated material. The authors found no significant difference in identification performance between real and simulated materials in an experiment with five materials. Yamamoto, Cros, Hashimoto, and Higuchi (2004) took a different approach and held the Peltier element at that temperature that, when the element was touched, would yield the same intermediate temperature as the simulated material would, given the thermal properties of the Peltier material, the finger, and the simulated material. Subjects were able to identify four materials fairly well (correct scores ranging from $53.3 \%$ to $90 \%$, with chance level at $25 \%$ ).

Although the object of these studies was not to measure discrimination thresholds, they do show that artificially displayed thermal stimuli are perceived fairly well. Since artificial stimuli can be generated at any desirable intensity, they enable us to measure precise discrimination thresholds in terms of thermal material parameters, at different values of these parameters. This would be a significant improvement upon threshold estimates based on measurements with (real or simulated) materials that are very widely spaced in terms of thermal parameters.

The aim of the present set of experiments was to measure precise thermal diffusivity thresholds by using controlled heat extraction. In Experiment 1, we studied the influence of the thermal time constant and temperature difference. Then, in Experiments 2 and 3, we split up the stimulus into two cues: a cue consisting of the temperature at the end of the stimulus display period (Experiment 2) and a cue consisting of the initial rate of temperature change (Experiment 3 ). This enabled us to study the contribution of these two separately. Finally, in Experiment 4, we checked the thresholds with a discrimination experiment with real materials.

\section{EXPERIMENT 1}

In this experiment, the thermal diffusivity discrimination threshold was measured in three different conditions.

\section{Stimulus Shape}

In the experiments with a Peltier heat pump discussed above, several methods were used for displaying thermal cues (prerecorded transient, static intermediate temperature). We used a method that came closest to the real thermal experience of touching an object. The general idea is that upon touch, there is an initial fast drop in temperature, followed by a continuous extraction of heat. Since there was already abundant research on the perception of tem- 
perature change of the kind that occurs in the first part, we focused instead on the second part, the continuous heat extraction. For this, a dynamic stimulus seemed to be the most natural. When a finger touches an object that has a lower temperature than the finger (e.g., room temperature) for an extended time, heat continuously flows from the finger into the object and disperses. The faster the heat disperses into the object, the cooler it will feel to the touch. If we consider the area of contact as a single point, we can use a spherically symmetric description. The way heat is distributed throughout the material is then described by the heat conduction equation in spherical coordinates:

$$
\frac{2}{r} \frac{d T(r, t)}{d r}+\frac{d^{2} T(r, t)}{d r^{2}}=\frac{\rho c}{k} \frac{d T(r, t)}{d t} .
$$

Here, $T(r, t)$ is the temperature at a distance $r$ from the point of contact and at time $t . \rho$ is the density of the material in $\mathrm{kg} / \mathrm{m}^{3}, c$ is the heat capacity of the material in $\mathrm{J} / \mathrm{K} / \mathrm{kg}$, and $k$ is the thermal conductivity coefficient in $\mathrm{W} / \mathrm{K} / \mathrm{m}$. The factor $\rho c / k$ in the right-hand side of the equation is the reciprocal of $\alpha \equiv k / \rho c$, which is known as the thermal diffusivity of the material and can be found in many reference books (e.g., Bejan, 1993). Since we are interested only in the temperature at the point of contact over time, we can ignore the spatial, $r$-dependent part and solve only the time-dependent part. A solution is

$$
T(t)=\left(T_{\mathrm{s}}-T_{\mathrm{e}}\right) \exp (-K \alpha t)+T_{\mathrm{e}},
$$

where $T_{\mathrm{s}}$ is the starting temperature, which will be an intermediate between skin temperature and room temperature, and $T_{\mathrm{e}}$ is the end temperature. $K$ is a constant that depends on the geometry of the simulated object. This exponential function has a time constant of $\tau=1 /(K \alpha)$. This means that in every time interval of length $\tau$, the temperature gets closer to the end temperature $T_{\mathrm{e}}$ by a factor of $1 / e$. For materials that have the same geometry, the factor $K$ is constant, and the thermal time constant $\tau$ is inversely proportional to the thermal diffusivity $\alpha$.

On the basis of this simple model of heat dispersion into the object, the temperature at the point of contact has the shape of an exponential decay. This adheres to Newton's (1701/1809) cooling law. It should be noted that this model does not take into account effects such as extra heat supply from the bloodstream in the finger, but it is to be expected that these effects cause only an offset in the cooling curve and do not significantly change the basic exponential shape.

In addition to the theoretical derivation above, there is also experimental evidence for this exponential shape. The temperature of the finger follows this profile until it freezes when cooled by cold air (Molnar, 1971). Temperature profiles resulting from cooling by different kinds of fabric show similar shapes (Schneider \& Holcombe, 1991). Cooling curves for the hand holding objects of six different materials could also be very well described by exponential functions (Havenith, van de Linde, \& Heus, 1992). Similar measurements were performed by Geng, Holmér, and Coldsurf Research Group (2001), yielding similar patterns. The measurements of finger temperature in Yamamoto et al. (2004) also seem to have this shape, especially at the lowest contact force of 200 gf. A curve measured at an even lower force of $100 \mathrm{gf}$ would probably have resembled an exponential decay even more. Finally, Sarda, Deterre, and Vergneault (2004) made a numerical model of a finger touching a surface. They also measured the temperature behavior using an artificial finger with thermal sensors. The profiles for the calculated and measured temperatures can be well approximated by exponential functions.

For these reasons, using an exponential function is the best choice when one wants to simulate the thermal aspect of touching a material. This has the added advantage that the starting and asymptotal end temperatures can be held constant, while only the rate of cooling between these temperatures is varied. Thus, the stimulus is not the amount of temperature change, but the time constant of the decay. This can be attained by using a temperature profile in accordance with Equation 2, in which the thermal diffusivity $\alpha$ is varied. The question we would like to answer with an experiment is the following: How well can subjects discriminate between (simulated) materials with different values of $\alpha$ ?

\section{Method}

Subjects. Twelve paid subjects, 2 male and 10 female university students, participated in the experiment. Their ages ranged from 17 to 26 years (average, 21.9). Young subjects were used in order to avoid decline in sensory function with age (Stevens \& Choo, 1998). All were strongly right-handed according to Coren's (1993) test.

Apparatus. To provide the heat extraction stimuli, a device was designed that was called a transient temperature threshold test thing (T5). It consisted of a Peltier element, a semiconductor thermometer, and a touch contact, as is shown in Figure 1. The Peltier element (Melcor PolarTEC PT8-12-40) was covered by a housing that left the touch contact exposed. The touch contact was a $4-\mathrm{cm}^{2}$ goldcoated copper square. Air was forced through the heat sink by a fan. The thermometer (Dallas Semiconductor DS600, accuracy better than $\pm 0.5^{\circ} \mathrm{C}$ ) and touch contact were read out by a computer, which also controlled the power supply that was connected to the Peltier element. The temperature was regulated by a software PID controller. When the touch contact was activated, the device provided a preprogrammed temperature transient. In this manner, temperature profiles that followed an exponential decay, as illustrated in Figure 2, could be presented to the subject. Because the rate of temperature change of an object cannot change instantaneously, there is some deviation of the rendered curves from the nominal curves at the start of the stimulus - in particular, with the shortest time constants. The mini-

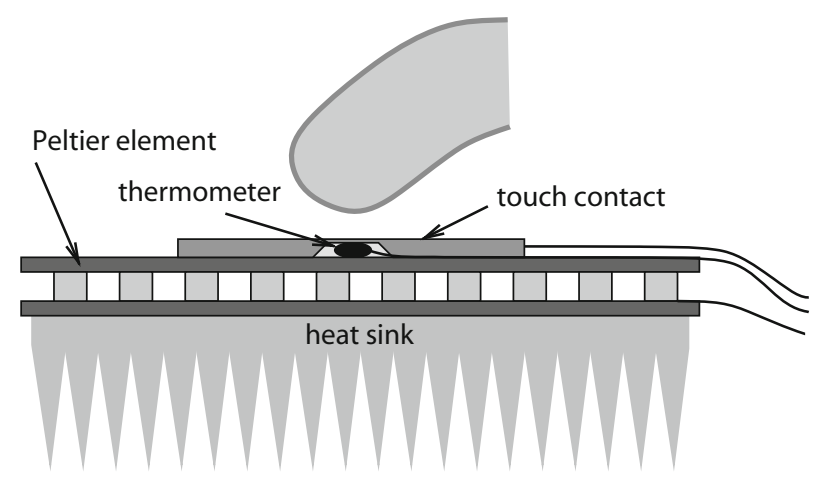

Figure 1. Schematic drawing of the setup (not to scale). 


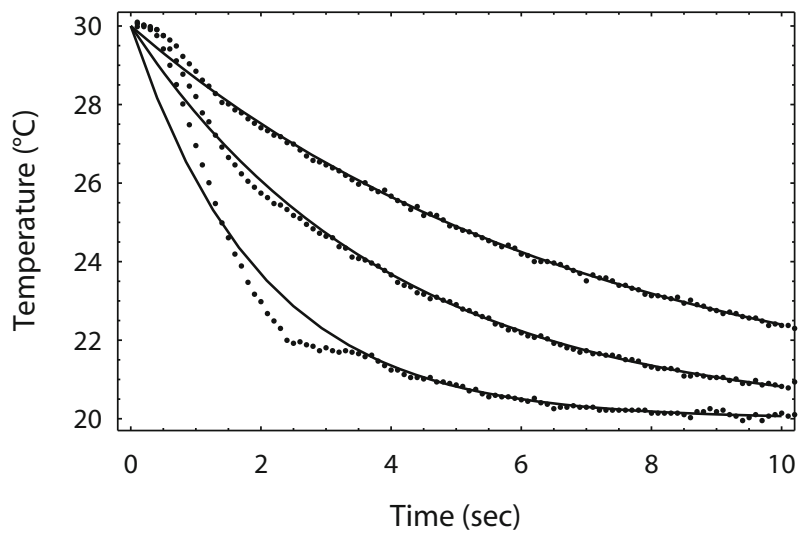

Figure 2. Examples of transient temperature profiles used as stimuli in Experiment 1, with time constants of 2, 4, and 7 sec. The solid lines are the nominal curves, and the dots are the actual curves.

mum time constant for which this deviation was deemed acceptable was $2 \mathrm{sec}$. Over all stimuli, the RMS deviation was $0.16^{\circ} \mathrm{C}$.

The whole setup was positioned on a digital weight scale that was read out by the same computer. The force used by the subject was displayed to him/her by an analogue indicator on a computer screen. This enabled the subject to maintain a constant and reproducible touching force.

Procedure. The experiment consisted of a two-alternative forced choice task in which, on each trial, the subjects had to choose, from two stimuli, the one that cooled faster. The stimuli were exponential temperature profiles as described by Equation 2. Each trial consisted of a standard and a comparison stimulus. Within the bounds of the desired accuracy, the T5 was limited to a shortest time constant of $\tau=$ $2 \mathrm{sec}$. On the basis of pilot experiments, the range for threshold measurements was chosen to be $10 \mathrm{sec}$ wide - that is, from 2 to $12 \mathrm{sec}$. Three conditions were selected for the experiment. The base condition was a standard stimulus in the center of the range, with a time constant of $\tau=7 \mathrm{sec}$ and comparison stimuli for which $\tau=2,4,6,8,10$, and $12 \mathrm{sec}$. The difference between starting and asymptotal end temperatures was $T_{\mathrm{s}}-T_{\mathrm{e}}=10^{\circ} \mathrm{C}$. There was a slow condition with the same temperature difference, a standard for which $\tau=14 \mathrm{sec}$, and comparisons for which $\tau=4,8,12,16,20$, and $24 \mathrm{sec}$. The third condition had the same time constants as the base condition but a temperature difference of $5^{\circ} \mathrm{C}$. The asymptotal end temperature in all cases was room temperature, which varied between $20.0^{\circ} \mathrm{C}$ and $22.8^{\circ} \mathrm{C}$. This was chosen because the rest of the subject's body was exposed to this temperature and, therefore, it was a natural reference point.

The subject started with his/her hand in an insulated box, which was kept at the starting temperature. $\mathrm{He} / \mathrm{she}$ was asked to put the hand flat with the palm down on a block that was in the box. In this way, the index finger was in contact with a surface that was kept at the starting temperature. At a signal from the experimenter, he/ she placed the right index finger on the T5, which started the temperature transient. The subject adjusted the touching force to $100 \mathrm{~g}$ $(0.98 \mathrm{~N})$ with the help of the analogue indicator. Tests indicated that the subjects were able to exert a force that was constant within $\pm 10 \%$. After $10 \mathrm{sec}$, the experimenter gave another signal, and the subject re-placed his/her hand into the insulated box, and the $\mathrm{T} 5$ was prepared for the second stimulus. This was presented in the same way, and after feeling it, the subject had to choose which stimulus had cooled faster. Each pair of stimuli was presented 10 times, half with first the standard and then the comparison and half the other way around. The 60 trials per condition were presented in random order. The three conditions were tested in different sessions of about $40 \mathrm{~min}$ each. The sessions took place either on different days or with at least $1 \mathrm{~h}$ of rest in between, but there were never more than two sessions per day. For the order in which the conditions were tested, all possible different permutations were used twice.

Although the subjects held their hand in a box kept at a constant temperature between trials, differences in "natural" skin temperature might have had an effect on thermal perception. To check for this, the skin temperature was measured before the start of each session. The subjects held the probe of a digital thermometer between their thumb and index finger until the readout had stabilized.

As a further check, for 6 of the subjects, the skin temperature at the end of each session was also measured. In the base condition, the RMS deviation of their skin temperature from the starting temperature at which the insulated box was kept was $2.5^{\circ} \mathrm{C}$. For comparison, the standard deviation over subjects of their skin temperatures at the start of the sessions was $4.4^{\circ} \mathrm{C}$. This indicates that the insulated box did a fair job of controlling skin temperature between trials.

In order to be able to assess the effect of the size of the contact area, the fingertip was photographed. Subjects pressed down on a transparent surface with the same force as that used in the experiment. A picture was then taken from the other side, using a digital video camera and a frame grabber in the computer. The size of the contact area could then be estimated by counting the pixels that exceeded a whiteness threshold.

Analysis. The number of times that the standard stimulus was perceived to cool faster was plotted as a function of the difference between the standard and the comparison stimulus. In Figure 3, a representative example of such a plot is shown. To this data, cumulative Gaussian functions were fitted using a maximum likelihood method. The $\sigma$ of these functions, corresponding to the $84 \%$ level, was taken as the threshold.

\section{Results}

In one condition, two subjects (J.G. and M.N.) had a very high threshold that was out of range for the stimulus set used. Therefore, these conditions were remeasured for those subjects. The measured thresholds for the 12 subjects are shown in Figure 4. Thresholds ranged from 1,702 to $4,483 \mathrm{msec}$ in the base condition and were generally higher in the other conditions. As compared with the base condition with a standard time constant of $7 \mathrm{sec}$, the average threshold doubled in the slow condition, with a standard time constant of $14 \mathrm{sec}$. For these conditions, which have a temperature difference between the start and end of the stimulus of

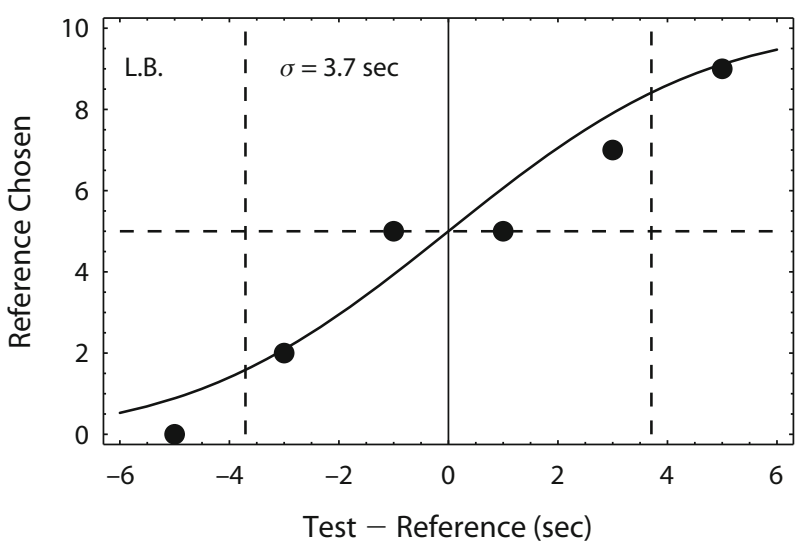

Figure 3. Representative example of the data from Experiment 1 for Subject $L$.B. in the $5^{\circ} \mathrm{C}$ temperature difference condition (dots), with a fitted function (solid line). The vertical dashed lines indicate the positions of $\pm \sigma$ of the cumulative Gaussian function. The horizontal dashed line indicates chance level. 


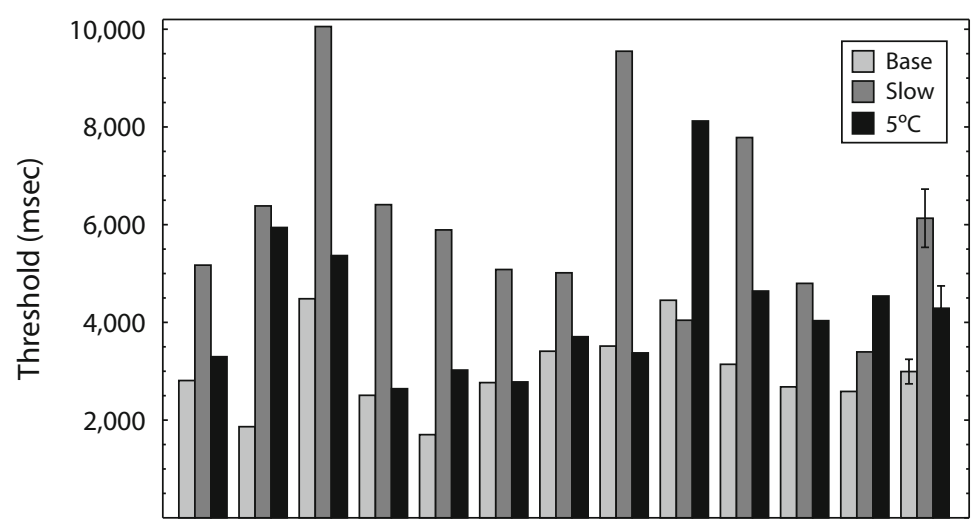

C.B. C.C. D.K. H.H. J.G. K.K. L.B. M.B. M.N. M.V. R.N. S.B. Mean

Figure 4. Thresholds of all subjects in the three conditions, plus the averages over subjects. The error bars for the averages indicate the standard errors of the sample means.

$10^{\circ} \mathrm{C}$, the threshold is a constant fraction of $43 \% \pm 13 \%$ of the standard time constant. Since the thermal diffusivity $\alpha$ is inversely proportional to the thermal time constant, the threshold is also $43 \%$ of the thermal diffusivity.

Thresholds were, on average, a factor of 1.4 higher in the $5^{\circ} \mathrm{C}$ temperature difference condition than in the base condition. However, some subjects (H.H., K.K., L.B., and M.B.) showed thresholds that were identical to those in the base condition. An ANOVA with repeated measures showed a significant effect of condition $[F(1.3,14.4)=$ $13.6, p=.0013]$. A Bonferroni-corrected post hoc analysis showed that the base and slow conditions were significantly different ( $p=.00055)$, but the $5^{\circ} \mathrm{C}$ condition was not significantly different from the slow condition $(p=$ $.11)$. The difference between the base and $5^{\circ} \mathrm{C}$ conditions was significant ( $p=.022)$.

Skin temperatures in the base condition ranged from $20.3^{\circ} \mathrm{C}$ to $33.4^{\circ} \mathrm{C}$. There was no correlation with discrimination threshold (Pearson's $r=-.13$, two-tailed $p=.69$ ). The size of the contact area ranged from 88 to $178 \mathrm{~mm}^{2}$. Here, also, no correlation with the thresholds was found $(r=.32, p=.31)$.

\section{Discussion}

Because of the asymptotic character of Equation 2, the temperature of the stimulus never quite reached the "end" temperature $T_{\mathrm{e}}$. The difference between $T_{\mathrm{e}}$ and the temperature at the end of the display period of $10 \mathrm{sec}$ depended on the time constant of the stimulus. For instance, for the stimuli in the base condition, this temperature difference ranged between $0.07^{\circ} \mathrm{C}$ and $4.3^{\circ} \mathrm{C}$. It could be suggested that these different temperatures at the end of the stimulus display period were used as a cue for discrimination, instead of or in addition to the cue of cooling rate. This would mean that the subject would base his/her judgment on absolute temperature discrimination, rather than on transient temperature discrimination. That is to say, the subjects would try to discriminate between the absolute temperatures of the subsequent stimuli at the end of their display period. However, if this were the case, the thresholds in the $5^{\circ} \mathrm{C}$ condition would be twice as high as those in the base condition, because the temperature differences at the end of the display period in the $5^{\circ} \mathrm{C}$ condition were half of those in the base condition. ${ }^{1}$ From the results shown in Figure 4, it is clear that this was not the case. The average threshold did go up in the $5^{\circ} \mathrm{C}$ condition, as compared with the base condition, but not by a factor of two. In fact, for 5 of the subjects, the thresholds hardly went up (C.B.) or went up not at all (H.H., K.K., L.B., and M.B.). This unchanged threshold could be expected if the judgments were based on the heat extraction rate: Although the absolute temperature difference was halved, the relative difference in heat extraction rate between the two stimuli in a trial remained the same. Therefore, it is unlikely that this task was dominated by absolute temperature discrimination.

Still, it is imaginable that absolute temperature perception does play a role in the discrimination of transient temperature profiles. The discrimination could then be based on a combination of two cues: the initial slope of the temperature change and the absolute temperature difference at the end of the display period. In order to assess the role of these two cues, two experiments were performed in which these cues were separated. In Experiment 2, the absolute temperature discrimination threshold was measured, whereas in Experiment 3, transient temperature profiles were used that had identical end temperatures, eliminating the cue of temperature difference at the end of the display period.

\section{EXPERIMENT 2}

In this experiment, the absolute temperature discrimination threshold was compared with the temperature differences at the end of the display period in the previous experiment. Because published temperature discrimination values were measured under circumstances that were very different from those in Experiment 1, they could not be used to do the comparison. Instead, we measured the temperature discrimination threshold in the same circumstances as those for the base condition in the previous experiment. 


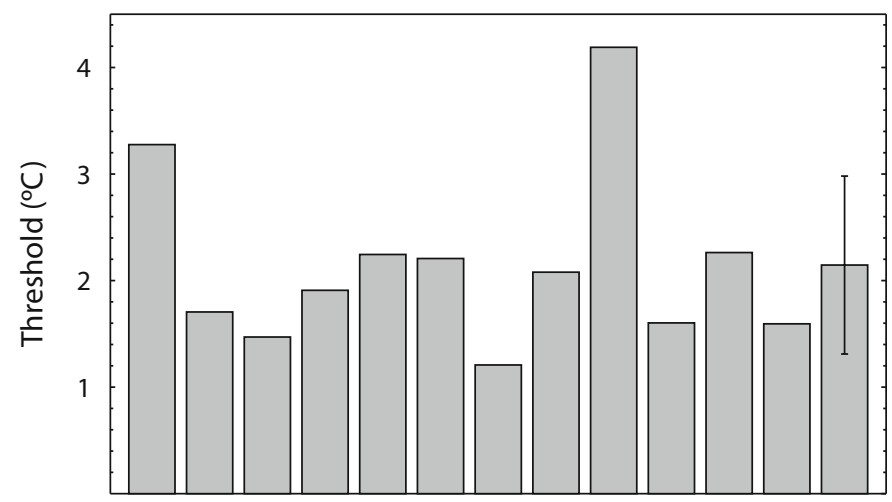

B.B. B.H. B.S. D.W. J.B. M.W. M.J. M.D. M.K. M.P. R.V. T.K. Mean

Figure 5. Absolute temperature discrimination thresholds for 12 subjects and their average. The error bar for the average indicates the standard deviation for the sample.

\section{Method}

Subjects. Twelve new subjects participated in the experiment. They were doctoral students in the physics department, 7 male and 5 female. One was left-handed. Their ages ranged from 24 to 29 years (average, 27.3).

Procedure. The setup and procedure were identical to those in Experiment 1, but this time, not transient but constant temperatures were displayed. The standard temperature of $22.4^{\circ} \mathrm{C}$ was compared with comparison temperatures of $20^{\circ} \mathrm{C}, 21^{\circ} \mathrm{C}, 22^{\circ} \mathrm{C}, 23^{\circ} \mathrm{C}, 24^{\circ} \mathrm{C}$, and $25^{\circ} \mathrm{C}$. The subject felt one stimulus for $1-2 \mathrm{sec}$, then placed his/her hand for $10 \mathrm{sec}$ in the insulated box kept at $30^{\circ} \mathrm{C}$, and then felt the second stimulus. This short exposure was chosen because the intention was to see whether the subjects could have used the end temperature in the profiles of Experiment 1 for discrimination, and those end temperatures were also available only for a short duration. The task was to indicate which temperature was the lower. There were 10 trials per comparison stimulus, totaling 60 trials per subject.

\section{Results and Discussion}

Cumulative Gaussian psychometric curves were fitted to the data in the same way as that in Experiment 1. The results are shown in Figure 5. The average threshold was $2.1^{\circ} \mathrm{C} \pm 0.8^{\circ} \mathrm{C}$. We can now compare this temperature threshold with the time constant threshold measured in the base condition of Experiment 1. In order to do so, the threshold has to be transformed from a time constant to a temperature. Through Equation 2, each value of the asymptotal end temperature $T_{\mathrm{e}}$ is related to a value of the time constant $\tau$. We can use the local slope of this relationship to convert a time constant interval (such as a discrimination threshold) to a temperature interval. This local slope is given by the derivative of Equation 2 with respect to $\tau$, at the end of the display period $(t=10 \mathrm{sec})$. When multiplied by this slope, the time constant threshold expressed in terms of temperature is $1.5^{\circ} \mathrm{C} \pm 0.4^{\circ} \mathrm{C}$. This is significantly smaller than the temperature threshold (one-tailed unpaired $t$ test, $t=2.5, p=.0098$ ). We use a one-tailed test because our hypothesis is that the threshold can be the same or higher but never lower, since the stimulus in this experiment contained less information than the stimulus in Experiment 1. Thus, the measured time constant threshold could never be attained if the discrimination were based solely on the temperature difference at the end of the display period. The temperature discrimination threshold is simply too high. From this, we can conclude that the time constant threshold is not dominated by absolute temperature discrimination.

\section{EXPERIMENT 3}

In order to assess the role of transient temperature perception in the discrimination of thermal time constants, a third experiment was performed.

\section{Method}

Subjects. The experiment was conducted with 12 new subjects, 7 male and 5 female. They were university students ranging in age from 18 to 23 years (average, 20.8). They were paid for their participation. All but 1 were right-handed.

Procedure. Again, the setup, procedure, and analysis were the same as those in Experiment 1, but this time, cooling curves were displayed that had the same temperature at the end of the display period $\left(t=t_{\mathrm{e}}\right)$. These curves were described by

$$
T(t)=\left(T_{\mathrm{s}}-T_{\mathrm{e}}\right)\left(\frac{t_{\mathrm{e}}-t}{t_{\mathrm{e}}}\right)^{n}+T_{\mathrm{e}} .
$$

Here, $n$ is the parameter that describes the shape of the curve. With $n=t_{\mathrm{e}} / \tau$, the curves have the same initial slope as the exponential curves of Equation 2. Examples of these curves are shown in Figure 6. In this way, curves are formed that have the same starting and also the same end temperature and differ only in initial cooling rate. It should be noted that, unlike in Experiment 1, these curves are not based on the physical process of touching an object. The purpose of Experiment 3 was to see how well people can discriminate between cooling profiles when only transient temperature cues are available.

A temperature profile stimulus with $n=1.43$ was compared with comparison stimuli with $n=0.83,1.00,1.25,1.67,2.50$, and 5.00. These stimuli had the same initial slope as those used in the base condition in Experiment 1. The temperature difference was $10^{\circ} \mathrm{C}$, and the end temperature was room temperature. The stimulus duration was $10 \mathrm{sec}$. There were 10 trials per comparison stimulus, totaling 60 trials per subject. The subjects were asked to touch the contact area upon a signal from the experimenter and feel the temperature going down from warm to cool, until the experimenter gave a second signal. Then they re-placed their hand in the temperature-controlled box. After feeling a pair of stimuli, they indicated which of the two cooled more quickly during the first part of the 10 -sec display pe- 


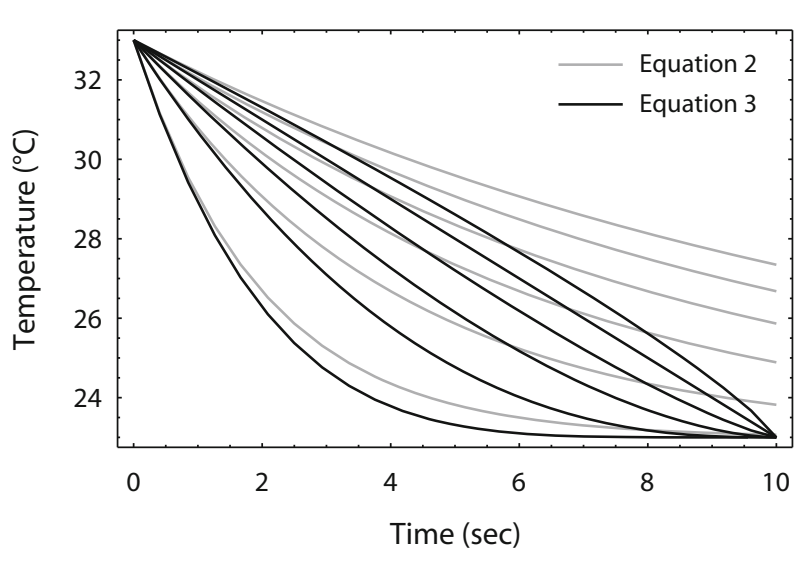

Figure 6. Examples of cooling curves used in Experiment 3 (black lines). Curves from Experiment 1 with the same initial slopes are shown in gray.

riod. It was important that they base their judgment on the first part of the temperature profile, because with all end temperatures being equal, the average cooling speed over the whole temperature profile was also the same for all the stimuli.

\section{Results and Discussion}

Two subjects were unable to perform the task. Their performance was at chance level over the whole stimulus range. When these 2 were subsequently tested with the task in the base condition in Experiment 1, their performance was also very different from that of the other subjects (thresholds of 4,556 and 5,417 msec, two to three standard deviations higher than the subjects in Experiment 1). It was decided that they must be unusually insensitive to temperature changes, and they were replaced with 2 others. The results of Experiment 3 are shown in Figure 7 , in terms of the time constant of the equivalent exponential temperature profile. The thresholds were generally higher than those in the base condition in Experiment 1 . On average, they were significantly higher (one- tailed unpaired $t$ test, $t=3.6, p=.00088$ ). Thus, without a temperature difference at the end of the display period as a source of information, discrimination performance was worse. This means that, in addition to the initial slope of the curves, the information at the end of the display period was also taken into account for the discrimination in Experiment 1.

\section{EXPERIMENT 4}

To confirm that the result from the first experiment with simulated thermal diffusivities would be valid in real life, a small experiment was performed with 16 colleagues. They were asked to discriminate between pairs of stimuli. One pair had a difference in thermal diffusivity that was below the threshold of $43 \%$, whereas the other pair had a difference that was above threshold. Three stimuli of $100 \times 100 \times 4 \mathrm{~mm}$ were used: copper, aluminum, and Plexiglas (acrylic glass). The thermal diffusivities of copper and aluminum are very close together $\left(1.07 \times 10^{-4}\right.$ and $8.42 \times 10^{-5} \mathrm{~m}^{2} / \mathrm{sec}$, respectively), whereas that of Plexiglas is much smaller $\left(1.1 \times 10^{-7} \mathrm{~m}^{2} / \mathrm{sec}\right)$ (Bejan, 1993). Thus, one pair of materials differs by a factor of 1.27 (i.e., 27\%), whereas the other differs by a factor of almost $10^{4}$. Ten pairs of copper/aluminum and 10 pairs of copper/Plexiglas were presented to the subject in random order. The subject used his/her index finger to feel the stimuli and had to decide which one felt colder. To prevent the stimuli from warming up, the subject could touch a stimulus only for less than $1 \mathrm{sec}$ at a time, but he/ she could go back and forth as often as desired. In the case of the copper/Plexiglas combination, the stimulus with the higher thermal diffusivity was chosen to be the colder on $93 \% \pm 18 \%$ of the trials. This was significantly different from the chance level of $50 \%(p=.022)$. With the copper/ aluminum combination, which would be expected to be below threshold for most people, the stimulus with the higher diffusivity was judged to be the colder on $37 \% \pm$ $24 \%$ of the trials. This was not significantly different from

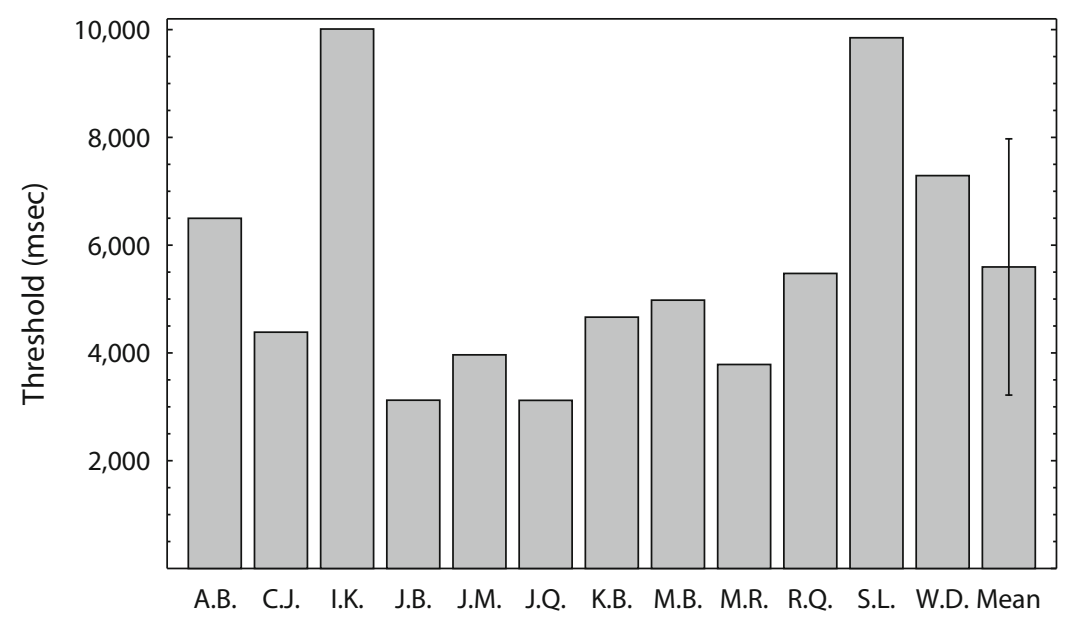

Figure 7. Discrimination thresholds for the cooling curves in Experiment 3 for 12 subjects and their average. The error bar for the average indicates the standard deviation for the sample. 
chance level $(p=.15)$. These significance levels were calculated by assuming a normal distribution based on the means and standard deviations for the scores. This distribution was cut off at $0 \%$ and $100 \%$ and was scaled so as to normalize the area between $0 \%$ and $100 \%$ to unity. Then the area of the $45 \%-55 \%$ interval around chance level was calculated. Thus, it was found that the pair with a thermal diffusivity difference above the measured threshold was successfully discriminated, whereas the pair with a difference below threshold was not.

\section{GENERAL DISCUSSION AND CONCLUSIONS}

The most important finding from these experiments is that in the conditions tested, the thermal time constant discrimination threshold was a constant fraction of $43 \%$ of the standard time constant when the temperature difference was $10^{\circ} \mathrm{C}$. The constancy of this fraction can be explained if we assume that the perception of heat extraction rate adheres to the Weber-Fechner law. $10^{\circ} \mathrm{C}$ is a normal difference between skin temperature $\left(\sim 30^{\circ} \mathrm{C}\right)$ and a typical room temperature $\left(\sim 20^{\circ} \mathrm{C}\right)$. Our results show that, under those circumstances, people can distinguish between materials solely on the basis of their thermal properties if their thermal time constants (and thus their thermal diffusivities $\alpha$, given the same geometry) are at least $43 \%$ apart. Consulting the table of thermal properties in, for example, Bejan (1993), we can see which materials are hard to distinguish if only thermal cues are available. For instance, the difference between copper and aluminum is below threshold, as is the difference between granite and marble. On the other hand, wood and plexiglas, or glass and steel, should be easy to discriminate. Perhaps surprisingly, cotton and brick are indistinguishable on the basis of heat extraction rate. But since these materials differ so much in other properties, this is of course impossible to verify experimentally.

We have verified experimentally that materials with a diffusivity difference below threshold are indeed difficult to discriminate. It was noted that when a difference in thermal diffusivity was not available as a cue (as was the case with the copper/aluminum combination), some subjects tried to discriminate using another thermal cue. When the subjects were allowed to touch the materials longer and with other parts of the hand, some of them reliably found aluminum to feel colder than copper, apparently using the difference in heat capacity as a cue. An object with a higher heat capacity warms up more slowly when touched continually. This might explain why some subjects felt aluminum, which has a higher heat capacity per unit volume, to be colder than copper, which has a higher thermal diffusivity. When the subjects were allowed to touch the materials only very briefly with just one finger, the materials probably did not warm up as much, and this effect was reduced.

The transient temperature profiles in Experiment 1 can be said to contain two major cues: different initial slopes and absolute temperature differences at the end of the display period. From Experiments 2 and 3, it became clear that when either cue is removed, the discrimination threshold goes up. This suggests that both cues are used for discrimination. There may be individual differences between subjects in this respect: For 5 of the subjects in Experiment 1, the threshold in the $5^{\circ} \mathrm{C}$ condition hardly went up, as compared with the base condition. Apparently, these 5 did not depend on information from the absolute temperature difference at the end of the display period.

The fact that the average threshold did not go up very much (a factor of only 1.4) when the temperature difference between the beginning and end of the stimulus was halved, as compared with the base condition (Experiment 1), indicates that the cue of thermal time constant is the more important one. We can conclude that for discrimination of materials on the basis of thermal properties, heat extraction rate is the most important cue (although by itself not sufficient, as was shown by Experiment 3), although the temperature difference after some time also plays a role (Experiment 2).

In earlier discrimination experiments (Ho \& Jones, 2006; Jones \& Berris, 2003), some materials with diffusivity differences above the threshold of $43 \%$ were not or poorly discriminated. Apparently, other factors that are not taken into account by the artificial extraction of heat affect the ability to discriminate between stimuli. In the present experiments, the same finger was used for both stimuli, whereas in the earlier experiments, two hands were used. This fact may also have played a role in this discrepancy. Having to compare two simultaneous sensations from different hands may be more difficult than comparing sequential sensations from the same hand. This was already observed by Weber $(1834 / 1996)$ in relation to temperature perception. We must conclude that the diffusivity thresholds in Experiment 1 are lower limits measured under ideal conditions.

The fact that "natural" skin temperature did not correlate with absolute threshold may have been caused by the small range of skin temperatures in Experiment 1. Some people have "natural" skin temperatures that are very different from the ones presented here. The data from this experiment are therefore insufficient to definitely rule out such a correlation.

A similar remark is valid for the correlation with contact area size. Since all the subjects used their fingertip to touch the stimuli, the variation in sizes was limited. It is not known what would happen to the thresholds if the whole hand could be used. In that case, a correlation with contact area size cannot be ruled out.

The absolute temperature thresholds from Experiment 2 are quite a bit higher than those found earlier (Johnson et al., 1973; Kenshalo et al., 1968; Stevens \& Choo, 1998). This can be ascribed to the very different circumstances in the present experiment. Between presentations, the subject had to remove his or her finger from the stimulator and put it in the insulated box, where it was warmed up again. This will have influenced the memory of the first stimulus of a pair. In addition, although the sequential presentation is probably an advantage, as compared with simultaneous presentation, due to the lower cognitive load associated with attending to only one stimulus at the same 
time, the long time $(15 \mathrm{sec})$ between presentations will have placed a higher load on memory than would occur with a shorter delay between presentations. Because we wanted to keep the circumstances as close as possible to those in Experiment 1, these thresholds are clearly not suitable for comparison with earlier findings measured under superior conditions.

In summary, we have begun to rigorously quantify the human ability for discriminating between thermal diffusivities of materials, and we have determined the relevant cues. Our experiment has provided a lower limit for the discrimination threshold that once more illustrates the high sensitivity of the human touch organ.

\section{AUTHOR NOTE}

This work was supported by a grant from the Netherlands Organisation for Scientific Research (NWO). Correspondence should be addressed to W. M. Bergmann Tiest, Helmholtz Institute, Utrecht University, Padualaan 8,3584CH Utrecht, The Netherlands (e-mail: w.m.bergmanntiest@ uu.nl).

\section{Note-Accepted by the previous editorial team,} when Thomas H. Carr was Editor.

\section{REFERENCES}

Аввотт, Е. (1914). The effect of adaptation on the temperature difference limen. Psychological Monographs, 16, 1-36.

BEJAN, A. (1993). Heat transfer. New York: Wiley.

Bergmann Tiest, W. M., \& Kappers, A. M. L. (2008). Thermosensory reversal effect quantified. Acta Psychologica, 127, 46-50.

Caldwell, D. G., \& Gosney, C. (1993). Enhanced tactile feedback (teletaction) using a multifunctional sensory system. In Proceedings of the International Conference on Robotics and Automation (Vol. 1, pp. 955-960). Los Alamitos, CA: IEEE Computer Society Press.

Coren, S. (1993). The left-hander syndrome: The causes and consequences of left-handedness. New York: Vintage.

Dyck, P. J., Curtis, D. J., BusheK, W., \& OfFord, K. (1974). Description of "Minnesota Thermal Disks" and normal values of cutaneous thermal discrimination in man. Neurology, 24, 325-330.

Geng, Q., Holmér, I., \& Coldsurf Research Group (2001). Change in contact temperature of finger touching on cold surfaces. International Journal of Industrial Ergonomics, 27, 387-391.

Green, B. G. (2004). Temperature perception and nociception. Journal of Neurobiology, 61, 13-29.

Greenspan, J. D., Roy, E. A., Caldwell, P. A., \& Faroog, N. S. (2003). Thermosensory intensity and affect throughout the perceptible range. Somatosensory \& Motor Research, 20, 19-26.

Havenith, G., van DE Linde, E. J. G., \& Heus, R. (1992). Pain, thermal sensation and cooling rates of hands while touching cold materials. European Journal of Applied Physiology \& Occupational Physiology, 65, 43-51.

Hilz, M. J., Glorius, S., \& Berić, A. (1995). Thermal perception thresholds: Influence of determination paradigm and reference temperature. Journal of the Neurological Sciences, 129, 135-140.

Ho, H.-N., \& JonEs, L. A. (2004). Material identification using real and simulated thermal cues. In Proceedings of the 26th Annual International Conference of the IEEE Engineering in Medicine and Biology Society (pp. 2462-2465). Los Alamitos, CA: IEEE Computer Society Press.

Ho, H.-N., \& JoNES, L. A. (2006). Contribution of thermal cues to material discrimination and localization. Perception \& Psychophysics, 68, $118-128$.

Ino, S., Shimizu, S., Odagawa, T., Sato, M., Takahashi, M., Izumi, T., \& Ifukube, T. (1993). A tactile display for presenting quality of materials by changing the temperature of skin surface. In Proceedings of the 2nd International Workshop on Robot and Human Communication (pp. 220-224). Los Alamitos, CA: IEEE Computer Society Press.

Johnson, K. O., Darian-Smith, I., \& LaMotte, C. (1973). Peripheral neural determinants of temperature discrimination in man: A correlative study of responses to cooling skin. Journal of Neurophysiology, 36, 347-370.

Jones, L. A., \& Berris, M. (2002). The psychophysics of temperature perception and thermal-interface design. In Proceedings of the 10th Symposium on Haptic Interfaces for Virtual Environment and Teleoperator Systems (pp. 137-142). Los Alamitos, CA: IEEE Computer Society Press.

JONES, L. A., \& BERRIS, M. (2003). Material discrimination and thermal perception. In Proceedings of the 11th Symposium on Haptic Interfaces for Virtual Environment and Teleoperator Systems (pp. 171178). Los Alamitos, CA: IEEE Computer Society Press.

KATZ, D. (1925). Der Aufbau der Tastwelt [The world of touch]. Leipzig: Barth.

Kenshalo, D. R., Holmes, C. E., \& Wood, P. B. (1968). Warm and cool thresholds as a function of rate of stimulus temperature change. Perception \& Psychophysics, 3, 81-84.

Molnar, G. W. (1971). Analysis of the rate of digital cooling. Journal de Physiologie, 63, 350-352.

Newton, I. (1809). A scale of the degrees of heat. In Philosophical transactions and collections to the end of the year 1720 abridged and disposed under general heads (Vol. 4, pp. 572-575). London: Royal Society. (English translation of Scala graduum caloris; original work published 1701)

Sarda, A., Deterre, R., \& Vergneault, C. (2004). Heat perception measurements of the different parts found in a car passenger compartment. Measurement, 35, 65-75.

Schneider, A. M., \& Holcombe, B. V. (1991). Properties influencing coolness to the touch of fabrics. Textile Research Journal, 61, 488-494.

Spray, D. C. (1986). Cutaneous temperature receptors. Annual Review of Physiology, 48, 625-638.

Stevens, J. C., \& Choo, K. K. (1998). Temperature sensitivity of the body surface over the life span. Somatosensory \& Motor Research, 15, 13-28.

TriTsCH, M. F. (1988). The veridical perception of object temperature with varying skin temperature. Perception \& Psychophysics, 43, 531540.

Weber, E. H. (1996). De tactu (H. E. Ross, Trans.). In H. E. Ross \& D. J. Murray (Eds.), E. H. Weber on the tactile senses (2nd ed.). Hove, U.K.: Taylor \& Francis. (Original work published 1834)

Yamamoto, A., Cros, B., Hashimoto, H., \& Higuchi, T. (2004). Control of thermal tactile display based on prediction of contact temperature. In Proceedings of the International Conference on Robotics and Automation (pp. 1536-1541). Los Alamitos, CA: IEEE Computer Society Press.

\section{NOTE}

1. The value of Equation 2 at the end of the display period $\left(t=t_{\mathrm{e}}\right)$ is $T=\left(T_{\mathrm{s}}-T_{\mathrm{e}}\right) \exp \left(-t_{\mathrm{e}} / \tau\right)+T_{\mathrm{e}}$. Its derivative with respect to $\tau$ is $d T /$ $d \tau=\left(T_{\mathrm{s}}-T_{\mathrm{e}}\right) t_{\mathrm{e}} \exp \left(-t_{\mathrm{e}} / \tau\right) / \tau^{2}$. The reciprocal is $d \tau / d T=\tau^{2} \exp \left(t_{\mathrm{e}} / \tau\right) /$ $\left(T_{\mathrm{s}}-T_{\mathrm{e}}\right) / t_{\mathrm{e}}$. This means that the difference in terms of time constant between two stimuli with a certain end temperature difference is inversely proportional to $T_{\mathrm{s}}-T_{\mathrm{e}}$. That is, when $T_{\mathrm{s}}-T_{\mathrm{e}}$ is halved, the difference between two time constants associated with a fixed end temperature difference doubles. Thus, if discrimination were based on end temperature differences, one would expect the time constant thresholds in the $5^{\circ} \mathrm{C}$ condition to be twice those in the base condition.

(Manuscript received May 31, 2006; revision accepted for publication November 10, 2008.) 